\title{
Fourier-Gauss Transforms of Bilinear Generating Functions for the Continuous $q$-Hermite Polynomials
}

\author{
M. K. Atakishiyeva* N. M. Atakishiyev ${ }^{\dagger \ddagger}$
}

CRM-2586

January 1999

*Facultad de Ciencias, UAEM, Apartado Postal 396-3, C.P. 62250, Cuernavaca, Morelos, Mexico

†Instituto de Matematicas, UNAM, Apartado Postal 273-3, C.P. 62210, Cuernavaca, Morelos, Mexico; natig@matcuer.unam.mx

${ }_{\ddagger}^{\ddagger}$ On leave from: Institute of Physics, Azerbaijan Academy of Sciences, H. Javid Prospekt 33, Baku 370143, Azerbaijan 


\begin{abstract}
The classical Fourier-Gauss transforms of bilinear generating functions for the continuous $q$-Hermite polynomials of Rogers are studied in detail. Our approach is essentially based on the fact that the $q$-Hermite functions have simple behaviour with respect to the Fourier integral transform with the $q$-independent exponential kernel.
\end{abstract}

\title{
Résumé
}

Les transformées de Fourier-Gauss pour les fonctions bilinéaires génératrices des qpolynômes d'Hermite de Rogers sont étudiées en détail. Notre approche est essentiellement basée sur le fait que les $q$-fonctions d'Hermite on un comportement simple par rapport à la transformée de Fourier intégrale avec le noyau exponentiel $q$-indépendant. 


\section{Introduction}

Bilinear generating functions (or Poisson kernels) are important tools for studying various properties of the corresponding families of orthogonal polynomials. For example, Wiener has used the bilinear generating function for the Hermite polynomials $H_{n}(x)$ in proving that the Hermite functions $H_{n}(x) \exp \left(-x^{2} / 2\right)$ are complete in the space $L_{2}$ over $(-\infty, \infty)$ and a Fourier transform of any function from $L_{2}$ belongs to the same space [1]. Also, it turns out that a particular limit value of the Hermite bilinear generating function reproduces the kernel $\exp (i x y)$ of Fourier transformation between two $L_{2}$ spaces. This idea was imployed for finding an explicit form of the reproducing kernel for the Kravchuk and Charlier functions in [2], whereas the case of the continuous $q$-Hermite functions was considered in [3].

It is clear that an appropriate $q$-analogue of the Fourier transform will be an essential ingredient of a completely developed theory of $q$-special functions. But the point is that the classical Fourier transform with the $q$-independent kernel turns out to be very useful in revealing close relations between some families of orthogonal $q$-polynomials [4,5], as well as among various $q$-extensions of the exponential function $e^{z}[6,7]$ and of the Bessel function $J_{\nu}(z)[8,9]$. One of the possible explanations of this remarkable circumstance is the simple Fourier-Gauss transformation property

$$
\frac{1}{\sqrt{2 \pi}} \int_{-\infty}^{\infty} e^{i r s-s^{2} / 2} x_{q}(s) d s=q^{1 / 4} x_{1 / q}(r) e^{-r^{2} / 2},
$$

enjoyed by the $q$-linear $x_{q}(s)=e^{i \kappa s}$ and, consequently, by the $q$-quadratic lattices $x_{q}(s)=\sin \kappa s$ or $x_{q}(s)=\cos \kappa s$ as well, where $q=\exp \left(-2 \kappa^{2}\right)$. It remains only to remind the reader that there exist a large class of polynomial solutions to the hypergeometric-type difference equation, which are defined in terms of these nonuniform lattices (see [10] for a review). Convinced of the power of classical Fourier transform, we wish to apply it for studying some additional properties of bilinear generating functions for the continuous $q$-Hermite polynomials of Rogers.

\section{Linear generating functions}

The continuous $q$-Hermite polynomials $H_{n}(x \mid q),|q|<1$, introduced by Rogers [11], are defined by their Fourier expansion

$$
H_{n}(x \mid q):=\sum_{k=0}^{n}\left[\begin{array}{l}
n \\
k
\end{array}\right]_{q} e^{i(n-2 k) \theta}, \quad x=\cos \theta
$$

where $\left[\begin{array}{l}n \\ k\end{array}\right]_{q}$ is the $q$-binomial coefficient,

$$
\left[\begin{array}{l}
n \\
k
\end{array}\right]_{q}=\frac{(q ; q)_{n}}{(q ; q)_{k}(q ; q)_{n-k}}
$$

and $(a ; q)_{0}=1,(a ; q)_{n}=\sum_{k=0}^{n-1}\left(1-a q^{k}\right), n=1,2,3, \ldots$, is the $q$-shifted factorial. These polynomials can be generated by the three-term recurrence relation

$$
2 x H_{n}(x \mid q)=H_{n+1}(x \mid q)+\left(1-q^{n}\right) H_{n-1}(x \mid q), \quad n \geq 0,
$$

with the initial condition $H_{0}(x \mid q)=1$. They have been found to enjoy many properties analogous to those known for the classical Hermite polynomials [11,12,13,14,15]. In particular, the Rogers 
generating function [11] for the $q$-Hermite polynomials has the form

$$
\sum_{n=0}^{\infty} \frac{t^{n}}{(q ; q)_{n}} H_{n}(\cos \theta \mid q)=e_{q}\left(t e^{i \theta}\right) e_{q}\left(t e^{-i \theta}\right), \quad|t|<1
$$

where the $q$-exponential function $e_{q}(z)$ and its reciprocal $E_{q}(z)$ are defined by

$$
e_{q}(z):=\sum_{n=0}^{\infty} \frac{z^{n}}{(q ; q)_{n}}=(z ; q)_{\infty}^{-1}, \quad E_{q}(z):=\sum_{n=0}^{\infty} \frac{q^{n(n-1) / 2}}{(q ; q)_{n}}(-z)^{n}=(z ; q)_{\infty} .
$$

It is often more convenient $(c f .[4,5,6,7])$ to make the change of variables $x=\cos \theta \rightarrow x_{q}(s)=\sin \kappa s$ in (2.4), which is equivalent to the substitution $\theta=\frac{\pi}{2}-\kappa s$. That is to say, one can represent (2.4) as

$$
g(s ; t \mid q):=\sum_{n=0}^{\infty} \frac{t^{n}}{(q ; q)_{n}} H_{n}(\sin \kappa s \mid q)=e_{q}\left(i t e^{-i \kappa s}\right) e_{q}\left(-i t e^{i \kappa s}\right), \quad|t|<1 .
$$

Observe that it is easy to verify $\left(2.4^{\prime}\right)$ directly, by substituting the explicit form of

$$
H_{n}(\sin \kappa s \mid q):=i^{n} \sum_{k=0}^{n}(-1)^{k}\left[\begin{array}{l}
n \\
k
\end{array}\right]_{q} e^{i(2 k-n) \kappa s}
$$

into it and interchanging the order of summations with respect to the indices $n$ and $k$.

The advantage of such a parametrization $x=\cos \theta=\sin \kappa s$ is that it actually incorporates both cases of the parameter $q$ : $0<|q|<1$ and $|q|>1$. Indeed, to consider the case when $|q|>1$ one may introduce the continuous $q^{-1}$-Hermite polynomials $h_{n}(x \mid q)$ as [16]

$$
h_{n}(x \mid q):=i^{-n} H_{n}\left(i x \mid q^{-1}\right) \text {. }
$$

The corresponding linear generating function for these polynomials is [17]

$$
\sum_{n=0}^{\infty} \frac{q^{n(n-1) / 2}}{(q ; q)_{n}} t^{n} h_{n}(\sinh \kappa s \mid q)=E_{q}\left(t e^{-\kappa s}\right) E_{q}\left(-t e^{\kappa s}\right)
$$

where $\left(c f .\left(2.1^{\prime}\right)\right)$

$$
h_{n}(\sinh \kappa s \mid q)=\sum_{k=0}^{n}(-1)^{k}\left[\begin{array}{l}
n \\
k
\end{array}\right]_{q} q^{k(k-n)} e^{(n-2 k) \kappa s} .
$$

From the inversion identity

$$
\left(q^{-1} ; q^{-1}\right)_{n}=(-1)^{n} q^{-n(n+1) / 2}(q ; q)_{n}, \quad n=0,1,2, \ldots,
$$

and the transformation property of the $q$-exponential functions (2.5) [18]

$$
e_{1 / q}(z)=E_{q}(q z)
$$

it follows that

$$
g\left(s ; t \mid q^{-1}\right)=\sum_{n=0}^{\infty} \frac{q^{n(n+1) / 2}}{(q ; q)_{n}}(-i t)^{n} h_{n}(\sinh \kappa s \mid q)=E_{q}\left(i q t e^{\kappa s}\right) E_{q}\left(-i q t e^{-\kappa s}\right) .
$$


The generating function $g\left(s ; i q^{-1} t \mid q^{-1}\right)$ thus coincides with the left-hand side of (2.7), so that (2.10) reproduces $(2.7)$.

Examination of equations $\left(2.4^{\prime}\right)$ and (2.10) reveals that the transformation of $q \rightarrow 1 / q$ actually provides a reciprocal to the $\left((2.4)^{\prime}\right)$ function

$$
g^{-1}(s ; t \mid q)=g\left(-i s ; q^{-1} t \mid q^{-1}\right)
$$

There is a second linear generating function

$$
f(s ; t \mid q):=\sum_{n=0}^{\infty} \frac{q^{n^{2} / 4}}{(q ; q)_{n}} t^{n} H_{n}(\sin \kappa s \mid q)=E_{q^{2}}\left(q t^{2}\right) \mathcal{E}_{q}(\sin \kappa s ; t)
$$

for the continuous $q$-Hermite polynomials $[19,6]$. The $q$-exponential function $\mathcal{E}_{q}(x ; t)$ in $(2.12)$ is defined by [19]

$$
\mathcal{E}_{q}(\sin \kappa s ; t):=e_{q^{2}}\left(q t^{2}\right) E_{q^{2}}\left(t^{2}\right) \sum_{n=0}^{\infty} \frac{q^{n^{2} / 4}}{(q ; q)_{n}}(-i t)^{n}\left(q^{\frac{1-n}{2}} e^{-i \kappa s},-q^{\frac{1-n}{2}} e^{i \kappa s} ; q\right)_{n},
$$

where $\left(a_{1}, \ldots, a_{k} ; q\right)_{n}=\prod_{j=1}^{k}\left(a_{j} ; q\right)_{n}$ is the conventional contracted notation for the multiple $q$ shifted factorials [20]. It is also expressible as a sum of two ${ }_{2} \phi_{1}$ basic hypergeometric series, i.e.,

$$
\begin{aligned}
\mathcal{E}_{q}(\sin \kappa s ; t)=e_{q^{2}}\left(q t^{2}\right) E_{q^{2}}\left(t^{2}\right)\left[{ }_{2} \phi_{1}\left(q e^{2 i \kappa s}, q e^{-2 i \kappa s} ; q ; q^{2}, t^{2}\right)\right. & \\
& \left.+\frac{2 q^{1 / 4} t}{1-q} \sin \kappa s_{2} \phi_{1}\left(q^{2} e^{2 i \kappa s}, q^{2} e^{-2 i \kappa s} ; q^{3} ; q^{2}, t^{2}\right)\right] .
\end{aligned}
$$

Introduced in [19] and further explored in [6,7,21], this $q$-analogue of the exponential function $e^{s t}$ on the $q$-quadratic lattice $x_{q}(s)=\sin \kappa s$ enjoys the property [19]

$$
\mathcal{E}_{1 / q}(x ; t)=\mathcal{E}_{q}\left(x ;-q^{1 / 2} t\right) .
$$

Therefore, as follows from (2.6), (2.8) and (2.12),

$$
f\left(s ; t \mid q^{-1}\right)=\sum_{n=0}^{\infty} \frac{q^{n^{2} / 4}}{(q ; q)_{n}}\left(-i q^{1 / 2} t\right)^{n} h_{n}(\sinh \kappa s \mid q)=e_{q}\left(q t^{2}\right) f\left(i s ;-q^{1 / 2} t \mid q\right) .
$$

Equating coefficients of like powers of the parameter $t$ on both sides of (2.16), we find the relation

$$
h_{n}(x \mid q)=i^{-n} \sum_{k=0}^{[n / 2]} \frac{\left(q^{-n} ; q\right)_{2 k}}{(q ; q)_{k}} q^{k(n-k+1)} H_{n-2 k}(i x \mid q)
$$

between the $q$-Hermite and $q^{-1}$-Hermite polynomials, in which $[n / 2]$ stands for the greatest integer not exceeding $n / 2$. In view of the definition (2.6), an alternate form of this expression is

$$
H_{n}\left(x \mid q^{-1}\right)=\sum_{k=0}^{[n / 2]} \frac{\left(q^{-n} ; q\right)_{2 k}}{(q ; q)_{k}} q^{k(n-k+1)} H_{n-2 k}(x \mid q)
$$


Once the relations (2.17) and (2.18) are established, they can be checked by a direct calculation using the explicit sums $(c f .(2.1))$

$$
\begin{aligned}
& H_{2 n}(x \mid q)=(-1)^{n}\left(q ; q^{2}\right)_{n 3} \phi_{2}\left(\begin{array}{c|c}
q^{-2 n},-e^{2 i \theta},-e^{-2 i \theta} & q^{2} ; q^{2} \\
q, 0 &
\end{array}\right. \\
& H_{2 n+1}(x \mid q)=(-q)^{-n}\left(q^{3} ; q^{2}\right)_{n} 2 x_{3} \phi_{2}\left(\begin{array}{c}
q^{-2 n},-q e^{2 i \theta},-q e^{-2 i \theta} \\
q^{3}, 0
\end{array} \mid q^{2} ; q^{2}\right),
\end{aligned}
$$

for the $q$-Hermite polynomials in terms of ${ }_{3} \phi_{2}$ basic hypergeometric series [6]. Indeed, replacing $q$ by $1 / q$ in $(2.19 \mathrm{a})$ and $(2.19 \mathrm{~b})$ results in

$$
\begin{aligned}
H_{2 n}\left(x \mid q^{-1}\right) & =q^{-n^{2}}\left(q ; q^{2}\right)_{n 3} \phi_{1}\left(q^{-2 n},-e^{2 i \theta},-e^{-2 i \theta} ; q ; q^{2}, q^{2 n+1}\right) \\
H_{2 n+1}\left(x \mid q^{-1}\right) & =q^{-n(n+1)}\left(q^{3} ; q^{2}\right)_{n} 2 x_{3} \phi_{1}\left(q^{-2 n},-q e^{2 i \theta},-q e^{-2 i \theta} ; q^{3} ; q^{2}, q^{2 n+1}\right) .
\end{aligned}
$$

Now substituting (2.19a) or (2.19b) into the right-hand side of (2.18) gives (2.20a) or (2.20b), respectively. Such a simple and elegant connection between the $q$-Hermite and $q^{-1}$-Hermite polynomials as (2.17) (or, equivalently, (2.18)) seems to deserve some attention.

Note that in the limit case when the parameter $q=\exp \left(-2 \kappa^{2}\right)$ tends to 1 (and, consequently, $\kappa \rightarrow 0$ ), we have

$$
\lim _{q \rightarrow 1^{-}} \kappa^{-n} H_{n}(\sin \kappa s \mid q)=\lim _{q \rightarrow 1^{-}} \kappa^{-n} h_{n}(\sinh \kappa s \mid q)=H_{n}(s),
$$

where $H_{n}(s)$ are the classical Hermite polynomials. The generating functions $\left(2.4^{\prime}\right)$ and (2.12) thus have the same limit value, i.e.,

$$
\lim _{q \rightarrow 1^{-}} g(s ; 2 \kappa t \mid q)=\lim _{q \rightarrow 1^{-}} f(s ; 2 \kappa t \mid q)=\sum_{n=0}^{\infty} \frac{t^{n}}{n !} H_{n}(s)=e^{2 s t-t^{2}} .
$$

As for the relations (2.17) or (2.18), letting $q$ tend to $1^{-}$in them, we obtain

$$
H_{n}(x)=i^{-n} n ! \sum_{k=0}^{[n / 2]} \frac{2^{k} H_{n-2 k}(i x)}{k !(n-2 k) !} .
$$

It is not hard to verify that this result agrees well with the known finite sum in powers of $x$

$$
H_{n}(x)=n ! \sum_{k=0}^{[n / 2]} \frac{(-1)^{k}(2 x)^{n-2 k}}{k !(n-2 k) !}
$$

for the classical Hermite polynomials.

To understand the group-theoretic origin of a particular classical generating function it is useful to know an appropriate differential equation for this function [22]. The continuous $q$-Hermite polynomials are solutions of the difference equation

$$
D_{q}(s) H_{n}(\sin \kappa s \mid q)=q^{-n / 2} \cos \kappa s H_{n}(\sin \kappa s \mid q)
$$

with an operator $D_{q}(s)$ defined by

$$
D_{q}(s):=\frac{1}{2}\left[e^{i \kappa s} e^{-i \kappa \partial_{s}}+e^{-i \kappa s} e^{i \kappa \partial_{s}}\right], \quad \partial_{s}=\frac{d}{d s} .
$$


To verify (2.25), apply the difference operator (2.26) to both sides of the Rogers generating function $\left(2.4^{\prime}\right)$ and then equate coefficients of the equal powers of the parameter $t$.

The difference equation (2.25) coincides in the limit of $q \rightarrow 1^{-}$with the second-order differential equation

$$
\left(\partial_{s}^{2}-2 s \partial_{s}+2 n\right) H_{n}(s)=0
$$

for the polynomials $H_{n}(s)$.

As a consequence of (2.25), the generating functions $\left(2.4^{\prime}\right)$ and (2.12) satisfy the same difference equation

$$
D_{q}(s) g(s ; t \mid q)=\cos \kappa s g\left(s ; q^{-1 / 2} t \mid q\right) \text {. }
$$

It may be of interest to note that the operator $D_{q}(s)$ is also well defined in the case of $|q|>1$. The explicit form of $D_{1 / q}(s)$ and its action on the generating functions (2.10) and (2.16) is readily obtained from (2.26) and (2.25), respectively, by the substitution $q \rightarrow q^{-1}(\kappa \rightarrow i \kappa)$.

In closing this section, we emphasize that the linear generating functions $g(s ; t \mid q)$ and $f(r ; t \mid q)$ are interrelated by a Fourier transformation with the standard exponential kernel $\exp ($ isr $)$, not involving $q$. Indeed, since the continuous $q$-Hermite and $q^{-1}$-Hermite polynomials are related to each other by the Fourier-Gauss transformation ( $c f$. formula (1.1))

$$
\frac{1}{\sqrt{2 \pi}} \int_{-\infty}^{\infty} H_{n}(\sin \kappa s \mid q) e^{i r s-s^{2} / 2} d s=i^{n} q^{n^{2} / 4} h_{n}(\sinh \kappa r \mid q) e^{-r^{2} / 2}
$$

one can multiply both sides of $(2.29)$ by $t^{n} /(q ; q)_{n}$ and sum over $n$ from zero to infinity. Taking into account (2.16), this gives

$$
\frac{1}{\sqrt{2 \pi}} \int_{-\infty}^{\infty} g(s ; t \mid q) e^{i r s-s^{2} / 2} d s=e_{q}\left(t^{2}\right) f(i r ; t \mid q) e^{-r^{2} / 2} .
$$

Observe also that substituting (2.17) into the right-hand side of (2.29), one may represent it alternatively as

$$
\frac{1}{\sqrt{2 \pi}} \int_{-\infty}^{\infty} H_{n}(\sin \kappa r \mid q) e^{-(r-s)^{2} / 2} d r=q^{n^{2} / 4} \sum_{k=0}^{[n / 2]} \frac{\left(q^{-n} ; q\right)_{2 k}}{(q ; q)_{k}} q^{k(n-k+1)} H_{n-2 k}(\sin \kappa s \mid q) .
$$

\section{Bilinear generating functions}

The $q$-Mehler formula (or the Poisson kernel) for the $q$-Hermite polynomials

$$
\sum_{n=0}^{\infty} \frac{t^{n}}{(q ; q)_{n}} H_{n}(\cos \theta \mid q) H_{n}(\cos \varphi \mid q)=E_{q}\left(t^{2}\right) e_{q}\left(t e^{i(\theta-\varphi)}\right) e_{q}\left(t e^{i(\varphi-\theta)}\right) e_{q}\left(t e^{i(\theta+\varphi)}\right) e_{q}\left(t e^{-i(\theta+\varphi)}\right)
$$

was originally derived by Rogers [11], its simple derivation is due to Bressoud [15]. As in the case of linear generating functions, it is more convenient to make the changes $x=\cos \theta \rightarrow \sin \kappa s=x_{q}(s)$ and $y=\cos \varphi \rightarrow \sin \kappa r=x_{q}(r)$ in (3.1), which are equivalent to substitutions $\theta=\frac{\pi}{2}-\kappa s$ and $\varphi=\frac{\pi}{2}-\kappa r$. In other words, one can represent (3.1) as

$$
\begin{aligned}
G(s, r ; t \mid q) & :=\sum_{n=0}^{\infty} \frac{t^{n}}{(q ; q)_{n}} H_{n}(\sin \kappa s \mid q) H_{n}(\sin \kappa r \mid q) \\
& =E_{q}\left(t^{2}\right) e_{q}\left(t e^{i \kappa(s-r)}\right) e_{q}\left(t e^{i \kappa(r-s)}\right) e_{q}\left(-t e^{i \kappa(s+r)}\right) e_{q}\left(-t e^{-i \kappa(s+r)}\right) \\
& =E_{q}\left(t^{2}\right) g\left(s ; i t e^{-i \kappa r} \mid q\right) g\left(s ;-i t e^{i \kappa r} \mid q\right),
\end{aligned}
$$


in accordance with the definition $\left(2.4^{\prime}\right)$ of the linear generating function $g(s ; t \mid q)$.

Notice that the bilinear generating functions (3.1) and (3.1') are closely connected with the Rogers linearization formula

$$
H_{m}(x \mid q) H_{n}(x \mid q)=\sum_{k=0}^{m \wedge n} \frac{(q ; q)_{m}(q ; q)_{n}}{(q ; q)_{m-k}(q ; q)_{n-k}(q ; q)_{k}} H_{m+n-2 k}(x \mid q)
$$

and its inverse $[11,15]$

$$
H_{n+n}(x \mid q)=(q ; q)_{m}(q ; q)_{n} \sum_{k=0}^{m \wedge n}(-1)^{k} q^{k(k-1) / 2} \frac{H_{m-k}(x \mid q) H_{n-k}(x \mid q)}{(q ; q)_{m-k}(q ; q)_{n-k}(q ; q)_{k}}
$$

where $m \wedge n:=\min \{m, n\}$. For instance, to verify $\left(3.1^{\prime}\right)$ one can substitute the explicit form $\left(2.1^{\prime}\right)$ for any one of the two $q$-Hermite polynomials in $\left(3.1^{\prime}\right)$ and use $\left(3.2^{\prime}\right)$ for the another one. Then the sum over the index $n$ in $\left(3.1^{\prime}\right)$ factorizes into a product of two linear generating functions of the type $\left(2.4^{\prime}\right)$ (see $\left(3.1^{\prime \prime}\right)$ ), which is multiplied by the $q$-exponential function $E_{q}\left(t^{2}\right)$.

As follows from (2.6), (2.8) and (2.9),

$$
\begin{aligned}
G\left(s, r ; t \mid q^{-1}\right) & =\sum_{n=0}^{\infty} \frac{q^{n(n+1) / 2}}{(q ; q)_{n}} t^{n} h_{n}(\sinh \kappa s \mid q)_{n}(\sinh \kappa r \mid q) \\
& =e_{q}\left(q t^{2}\right) E_{q}\left(q t e^{\kappa(s-r)}\right) E_{q}\left(q t e^{\kappa(r-s)}\right) E_{q}\left(-q t e^{\kappa(s+r)}\right) E_{q}\left(-q t e^{-\kappa(s+r)}\right) \\
& =e_{q}\left(q t^{2}\right) g\left(s ; i t e^{\kappa r} \mid q^{-1}\right) g\left(s ;-i t e^{-\kappa r} \mid q^{-1}\right) .
\end{aligned}
$$

This coincides with the $q$-Mehler formula for the $q^{-1}$-Hermite polynomials [17]

$$
\begin{aligned}
& \sum_{n=0}^{\infty} \frac{q^{n(n+1) / 2}}{(q ; q)_{n}} t^{n} h_{n}(\sinh \xi \mid q) h_{n}(\sinh \eta \mid q) \\
& \quad=e_{q}\left(q t^{2}\right) E_{q}\left(q t e^{\xi-\eta}\right) E_{q}\left(q t e^{\eta-\xi}\right) E_{q}\left(-q t e^{\xi+\eta}\right) E_{q}\left(-q t e^{-\xi-\eta}\right)
\end{aligned}
$$

upon identifying $\kappa s=\xi$ and $\kappa r=\eta$. It is worth noting that Ismail and Masson [17] have employed the Poisson kernel (3.4) to determine the large $n$ asymptotics of the $q^{-1}$-Hermite polynomials $h_{n}(x \mid$ $q)$.

Observe also the relation

$$
G^{-1}(s, r ; q t \mid q)=\left(1-q t^{2}\right) G\left(i s, i r ; t \mid q^{-1}\right),
$$

which follows from the $q$-Mehler formulae (3.2) and (3.3).

As a consequence of (2.6) and (2.29), the generating functions (3.1') and (3.3) are related to each other by the Fourier-Gauss transform in the variables $s$ and $r$, i.e.,

$$
G\left(s, r ; t \mid q^{-1}\right) e^{-\left(s^{2}+r^{2}\right) / 2}=\frac{1}{2 \pi} \int_{-\infty}^{\infty} G\left(u, v ; q^{\frac{1}{2}} t \mid q\right) e^{i(s u-r v)-\left(u^{2}+v^{2}\right) / 2} d u d v .
$$

The Fourier-Gauss transform (3.6) is equivalent to a particular case of Ramanujan's integral

$$
\frac{1}{\sqrt{\pi}} \int_{-\infty}^{\infty} e_{q}\left(a e^{2 i \kappa x}\right) e_{q}\left(b e^{-2 i \kappa x}\right) e^{2 i x y-x^{2}} d x=e_{q}(a b) E_{q}\left(-a q^{1 / 2} e^{2 \kappa y}\right) E_{q}\left(-b q^{1 / 2} e^{-2 \kappa y}\right) e^{-y^{2}}
$$


with a complex parameter [23, 24,25]. Indeed, using $\left(3.1^{\prime}\right)$ and substituting $s_{ \pm}=(s \pm r) / \sqrt{2}$ and $u_{ \pm}=(v \pm u) / \sqrt{2}$ leads to the separation of variables in the right-hand side of (3.6) and gives a product of two integrals with respect to $u_{+}$and $u_{-}$. Both of these independent integrals are of the type (3.7) with the equal parameters $a=b=q^{1 / 2} t$. One thus recovers the left-hand side of (3.6) with the generating function $G\left(s, r ; t \mid q^{-1}\right)$, defined in (3.3).

Two particular cases of the generating function $G(s, r ; t \mid q)$ are of interest for purposes of the use in the sequel. First of them is $G(s, 0 ; t \mid q)$. Since $H_{2 k}(0 \mid q)=(-1)^{k}\left(q ; q^{2}\right)_{k}, H_{2 k+1}(0 \mid q)=0$, and $(q ; q)_{2 k}=\left(q ; q^{2}\right)_{k}\left(q^{2} ; q^{2}\right)_{k}$, this function represents the sum

$$
G(s, 0 ; t \mid q)=\sum_{n=0}^{\infty} \frac{(-1)^{n} t^{2 n}}{\left(q^{2} ; q^{2}\right)_{n}} H_{2 n}(\sin \kappa s \mid q) .
$$

By the $q$-Mehler formula (3.2) this sum is equal to

$$
G(s, 0 ; t \mid q)=E_{q}\left(t^{2}\right) e_{q^{2}}\left(t^{2} e^{2 i \kappa s}\right) e_{q^{2}}\left(t^{2} e^{-2 i \kappa s}\right)
$$

on account of $e_{q}(z) e_{q}(-z)=e_{q^{2}}\left(z^{2}\right)$.

Similarly, since $h_{2 n}(0 \mid q)=(-1)^{n} q^{-n^{2}}\left(q ; q^{2}\right)_{n}$ and $h_{2 n+1}(0 \mid q)=0$, from (3.3) we have

$$
G\left(s, 0 ; t \mid q^{-1}\right)=\sum_{n=0}^{\infty} \frac{(-1)^{n} q^{n(n+1)}}{\left(q^{2} ; q^{2}\right)_{n}} t^{2 n} h_{2 n}(\sinh \kappa s \mid q)=e_{q}\left(q t^{2}\right) E_{q^{2}}\left(q^{2} t^{2} e^{2 \kappa s}\right) E_{q^{2}}\left(q^{2} t^{2} e^{-2 \kappa s}\right) .
$$

As a consequence of (2.25), the generating function $G(s, r ; t \mid q)$ satisfies the following difference equations

$$
\begin{aligned}
D_{q}(s) G(s, r ; t \mid q) & =\cos \kappa s G\left(s, r ; q^{-1 / 2} t \mid q\right), \\
D_{q}(s) D_{q}(r) G(s, r ; t \mid q) & =\cos \kappa s \cos \kappa r G\left(s, r ; q^{-1} t \mid q\right) .
\end{aligned}
$$

Observe that if the independent variables $s$ and $r$ are replaced by their linear combinations $s_{ \pm}=$ $(s \pm r) / \sqrt{2}$, then the product of difference operators $D_{q}(s)$ and $D_{q}(r)$ takes the form

$$
D_{q}(s) D_{q}(r)=\frac{1}{2}\left[D_{q^{2}}\left(s_{+}\right)+D_{q^{2}}\left(s_{-}\right)\right] .
$$

The integral transform (2.2), relating the continuous $q$-Hermite and $q^{-1}$-Hermite polynomials, suggests also the consideration of the "mixed" generating function

$$
F(s, r ; t \mid q)=\sum_{n=0}^{\infty} \frac{q^{n^{2} / 4}}{(q ; q)_{n}} t^{n} H_{n}(\sin \kappa s \mid q) h_{n}(\sinh \kappa r \mid q),
$$

which is different from the known functions $\left(3.1^{\prime}\right)$ and (3.3). Unlike $G(s, r ; t \mid q)$, the generating function $F(s, r ; t \mid q)$ is not symmetric in the variables $s$ and $r$. Instead, it has the following property

$$
F\left(s, r ; t \mid q^{-1}\right)=F\left(r, s ; q^{1 / 2} t \mid q\right) \text {. }
$$

Also, a relation between the "mixed" generating function (3.13) and linear generating functions $\left(2.4^{\prime}\right)$ and (2.12) is more complicated than (3.1"). Indeed, substitute the explicit form of the $q^{-1}$ Hermite polynomials $\left(2.6^{\prime}\right)$ into (3.13) and interchange the order of summations with respect to the 
indices $n$ and $k$. The subsequent use of the inverse (3.2') to the Rogers linearization formula (3.2) factors out the $q$-exponential function $E_{q}\left(-t^{2}\right)\left(c f .\left(3.1^{\prime \prime}\right)\right)$ and gives the following relations

$$
\begin{aligned}
F(s, r ; t \mid q) & =E_{q}\left(-t^{2}\right) \sum_{n=0}^{\infty} q^{n^{2} / 4} g_{n}\left(s ; t e^{-\kappa r} \mid q\right) f\left(s, q^{-n / 2} t e^{\kappa r} \mid q\right) \\
& =E_{q}\left(-t^{2}\right) \sum_{n=0}^{\infty} f_{n}\left(s,-t e^{-\kappa r} \mid q\right) f\left(s, q^{-n / 2} t e^{\kappa r} \mid q\right),
\end{aligned}
$$

where

$$
g_{n}(s ; t \mid q):=\frac{t^{n}}{(q ; q)_{n}} H_{n}(\sin \kappa s \mid q), \quad n=0,1,2, \ldots
$$

and $f_{n}(s ; t \mid q)=q^{n^{2} / 4} g_{n}(s ; t \mid q)$ are the partial linear generating functions of the first kind $\left(2.4^{\prime}\right)$ and of the second kind (2.12), respectively, i.e,

$$
g(s ; t \mid q)=\sum_{n=0}^{\infty} g_{n}(s ; t \mid q), \quad f(s ; t \mid q)=\sum_{n=0}^{\infty} f_{n}(s ; t \mid q) .
$$

From the relation (2.29) between the $q$-Hermite and $q^{-1}$-Hermite polynomials and the definition (3.13) it follows that the Fourier transform of $F(s, r ; t \mid q) \exp \left(-s^{2} / 2\right)$ is equal to

$$
\frac{1}{\sqrt{2 \pi}} \int_{-\infty}^{\infty} F(s, r ; t \mid q) e^{i s u-s^{2} / 2} d s=G\left(u, r ; i q^{-1 / 2} t \mid q^{-1}\right) e^{-u^{2} / 2} .
$$

In a like manner, the inverse Fourier transformation with respect to (2.29) yields

$$
\frac{1}{\sqrt{2 \pi}} \int_{-\infty}^{\infty} F(s, r ; t \mid q) e^{i r v-r^{2} / 2} d r=G(s, v ; i t \mid q) e^{-v^{2} / 2} .
$$

Since the explicit form of the bilinear generating function $G(u, v ; t \mid q)$ is given by the formulae (3.2) and (3.3) for the values $0<|q|<1$ and $|q|>1$ of the parameter $q$, respectively, the relations (3.17) and (3.18) lead to the two integral representations for $F(s, r ; t \mid q)$ of the form

$$
\begin{aligned}
F(s, r ; t \mid q) e^{-s^{2} / 2}=\frac{e_{q}\left(-t^{2}\right)}{\sqrt{2 \pi}} \int_{-\infty}^{\infty} e^{-i s u-u^{2} / 2} & E_{q}\left(i q^{1 / 2} t e^{\kappa(u-r)}\right) E_{q}\left(i q^{1 / 2} t e^{\kappa(r-u)}\right) \\
& \times E_{q}\left(-i q^{1 / 2} t e^{\kappa(u+r)}\right) E_{q}\left(-i q^{1 / 2} t e^{-\kappa(u+r)}\right) d u \\
F(s, r ; t \mid q) e^{-r^{2} / 2}=\frac{E_{q}\left(-t^{2}\right)}{\sqrt{2 \pi}} \int_{-\infty}^{\infty} e^{-i r v-v^{2} / 2} & e_{q}\left(i t e^{i \kappa(s-v)}\right) e_{q}\left(i t e^{i \kappa(v-s)}\right) \\
& \times e_{q}\left(-i t e^{i \kappa(s+v)}\right) e_{q}\left(-i t e^{-i \kappa(s+v)}\right) d v .
\end{aligned}
$$

Also, combining (3.17) with (3.18) shows that the Fourier-Gauss transform of $F(s, r ; t \mid q)$ in both independent variables $s$ and $r$ reproduces this generating function, i.e.,

$$
F(v, u ; t \mid q) e^{-\left(u^{2}+v^{2}\right) / 2}=\frac{1}{2 \pi} \int_{-\infty}^{\infty} F(s, r ; t \mid q) e^{i(s u-r v)-\left(s^{2}+r^{2}\right) / 2} d s d r
$$

Similar to $G(s, r ; t \mid q)$, two particular cases of $F(s, r ; t \mid q)$ are easily summed as products of $q$ exponential functions. The first one is $F(s, 0 ; t \mid q)$ and it coincides with $G(s, 0 ; t \mid q)$, given by (3.8) 
and (3.9). The second one is $F(0, r ; t \mid q)=G\left(r, 0 ; q^{-1 / 2} t \mid q^{-1}\right)$, where the function $G\left(r, 0 ; t \mid q^{-1}\right)$ is explicitly given in (3.10).

From (2.25) it follows at once that the mixed generating function (3.13) satisfies the difference equations in the variables $s$ and $r$ of the form

$$
\begin{aligned}
D_{q}(s) F(s, r ; t \mid q) & =\cos \kappa s F\left(s, r ; q^{-1 / 2} t \mid q\right), \\
D_{1 / q}(r) F(s, r ; t \mid q) & =\cosh \kappa r F\left(s, r ; q^{1 / 2} t \mid q\right) .
\end{aligned}
$$

In view of the property (3.14), these two equations are related to each other by the substitution $q \rightarrow q^{-1}$. Combining (3.21a) with (3.21b) gives the difference equation

$$
D_{q}(s) D_{1 / q}(r) F(s, r ; t \mid q)=\cos \kappa s \cosh \kappa r F(s, r ; t \mid q) \text {. }
$$

The authors are most grateful to the Centre de recherches mathématiques, Université de Montréal, and to Pavel Winternitz, in particular, for the hospitality extended to them during their visit to Montréal in December 1998-January 1999, when this work was completed. This research is partially supported by the UNAM-DGAPA project IN106595.

\section{References}

[1] N. Wiener. The Fourier Integral and Certain of Its Applications, Cambridge University Press, Cambridge, 1993.

[2] R. Askey, N. M. Atakishiyev and S. K. Suslov. Proceedings of the XV Workshop on High Energy Physics and Field Theory (Protvino, Russia, 6-10 July, 1992), eds. A. P. Samokhin and G. L. Rcheulishvili, pp. 140-144, IHEP, Protvino, 1995.

[3] R. Askey, N. M. Atakishiyev and S. K. Suslov. Symmetries in Science, VI, ed. B. Gruber, pp. 57-63, Plenum Press, New York, 1993.

[4] N. M. Atakishiyev and Sh. M. Nagiyev. Theor. Math. Phys., Vol. 98, No. 2, pp. 162-166, 1994.

[5] N. M. Atakishiyev and Sh. M. Nagiyev. J. Phys. A: Math. Gen., Vol. 27, No. 17, pp. L611-L615, 1994.

[6] N. M. Atakishiyev and P. Feinsilver. J. Phys. A: Math. Gen., Vol. 29, No. 8, pp. 1659-1664, 1996.

[7] N. M. Atakishiyev. J. Phys. A: Math. Gen., Vol. 29, No. 10, pp. L223-L227, 1996; J. Phys. A: Math. Gen., Vol. 29, No. 22, pp. 7177-7181, 1996.

[8] N. M. Atakishiyev. Proceedings of the 2nd Conference on "Symmetries and Integrability of Difference Equations" (University of Kent, Canterbury, England, 1-5 July, 1996), to appear.

[9] M. K. Atakishiyeva and N. M. Atakishiyev. J. Phys. A: Math. Gen., Vol. 30, No. 13, pp. L429-L432, 1997.

[10] N. M. Atakishiyev, M. Rahman and S. K. Suslov. Constructive Approximation, Vol. 11, No. 2, pp. 181-226, 1995. 
[11] L. J. Rogers. Proc. London Math. Soc., Vol. 24, pp. 171-179, 1893; Vol. 24, pp. 337-352, 1893; Vol. 25, pp. 318-343, 1894; Vol. 26, pp. 15-32, 1895.

[12] G. Szegö. Sitz. Preuss. Akad. Wiss., Phys. -Math. klasse, Vol. 19, pp. 242-252, 1926; reprinted in Collected Papers, ed. R. Askey, Vol. 1, pp. 795-805, Birkhäuser, Boston, MA, 1982.

[13] L. Carlitz. Ann. Matem. Pura Appl., Ser. 4, Vol. 41, pp. 359-373, 1955; Duke Math. J., Vol. 24, pp. 521-527, 1957.

[14] R. Askey and M. E. H. Ismail. Studies in Pure Mathematics, ed. P. Erdös, pp. 55-78, Birkhäuser, Boston, MA, 1983.

[15] D. M. Bressoud. Ind. Univ. Math. J., Vol. 29, No. 4, pp. 577-580, 1980.

[16] R. Askey. q-series and Partitions, ed. D. Stanton, IMA Volumes in Mathematics and Its Applications, pp. 151-158, Springer-Verlag, New York, 1989.

[17] M. E. H. Ismail and D. R. Masson. Trans. Amer. Math. Soc., Vol. 346, No. 1, pp. 63-116, 1994.

[18] H. Exton. q-Hypergeometric Functions and Applications, Ellis Horwood, Chichester, 1983.

[19] M. E. H. Ismail and R. Zhang. Adv. Math.,i Vol. 109, No. 1, pp. 1-33, 1994.

[20] G. Gasper and M. Rahman. Basic Hypergeometric Series, Cambridge University Press, Cambridge, 1990.

[21] S. K. Suslov. Methods and Applications of Analysis, Vol. 4, No. 1, pp. 11-32, 1997.

[22] L. Weisner. Pacific J. Math., Suppl. II, Vol. 5, pp. 1033-1039, 1955.

[23] S. Ramanujan. Collected Papers, eds. G. H. Hardy, P. V. Seshu Aiyar and B. M. Wilson, Cambridge University Press, Cambridge, 1927; reprinted by Chelsea, New York, 1959.

[24] R. Askey. Proc. Amer. Math. Soc., Vol. 85, No. 2, pp. 192-194, 1982.

[25] N. M. Atakishiyev and P. Feinsilver. Proceedings of the IV Wigner Symposium (Guadalajara, Mexico, 7-11 August, 1995), eds. N. M. Atakishiyev, T. H. Seligman and K. B. Wolf, pp. 406-412, World Scientific, Singapore, 1996. 